\title{
RADAR, A FRAMEWORK FOR AUTOMATED REPORTING
}

\author{
Antonia Azzini ${ }^{1}$, Nicola Cortesi ${ }^{1}$, Amir Topalovic $^{1}$ and Giuseppe Psaila ${ }^{2}$ \\ ${ }^{I}$ Consortium for the Technology Transfer - C2T, Via Marengo, 22 - Carate Brianza (MB) Italy \\ ${ }^{2}$ University of Bergamo, viale Marconi, 5 - Dalmine (BG) Italy
}

\begin{abstract}
Large companies and organizations periodically feed their information systems with large data flows. Apart from the classical operational activities, they are called to prepare aggregated reports to send to institutions and rating agencies. Unfortunately, organizations typically suffer for the lack of integrated data and for the lack of a standard data dictionary. The presented approach aims to tackle such a problem by building a "bridge" between employees that need to specify how to generate reports (on the basis of concepts and terms typical of the application domain) and the information system that stores the data to query and aggregate in order to automatically produce reports. The implemented framework, RADAR (Rich Advanced Design Approach for Reporting), moves from the notion of Operational Data Store, and it is posed in the middle between an ontology (of concepts and terms) and the actual operational (and relational) schema of source data. Then, in the defined schema allows for giving a high-level view of such source data, based on concepts described in the ontology for a specific application domain.
\end{abstract}

\section{KEYWORDS}

Knowledge Representation, Automation of Services, Data Integration, IT Services

\section{INTRODUCTION}

Current information system technology is able to store and process a huge amount of data. In case of large corporations or institutions (such as financial institutions, multinational manufacturers, central banks, etc.) information systems are fed with large data flows that are provided periodically (e.g., daily, weekly, and monthly). Database administrators and system integrators have been developing solutions to efficiently receive and manage these data flows, by optimizing loading processes as much as possible.

Apart from traditional operational activities, collected data are essential for reporting activities. In fact, regulatory bodies (such as central banks) and national offices of statistics as well as rating agencies ask companies and institutions to periodically deliver reports illustrating the current state of their business. The reasons are manifold: they can vary from the need to monitor trends of economy, to the need to provide markets with transparent information concerning the current economical state of companies. Nevertheless, companies might need to prepare reports for internal use, for example for auditing and for decision-making.

Typically, organizations suffer for the lack of integrated data and for the lack of a standard data dictionary; then, significant manual intervention to write queries and create reports is necessary (Browne, et al., 2019). Moreover, employees who are responsible for producing reports in general are not aware of technicalities concerning how data are stored and how they can be queried; consequently, they have to interact with technicians that also have to be experts in the application domain, so as to understand requests made by employees (these professional figures are very rare). Clearly, there is a gap between users and systems that can be resumed by the following questions: "What is the meaning of data? How could they be interpreted to meet the requirements? How could people, who are not database programmers, define reports?".

To further complicate the scenario, terms and concepts are often informally defined by international organizations; furthermore, the meaning can vary in specific communities. Specialists use a lot of complex interconnected concepts, including those defined by international standards and systems, developed to provide, e.g., global financial services. Clearly, a common comprehension of terms and their semantics is essential to prepare reports: knowledge about terms and data is crucial to define reports effectively and efficiently. 
In our research, we are trying to tackle the problem by building a bridge between employees that need to specify how to generate reports (based on concepts and terms typical of the application domain) and the information system that stores the data to be queried and aggregated in order to produce the reports. The result is $R A D A R$ (acronym for Rich Advanced Design Approach for Reporting), a novel software framework that moves from the notion of Operational Data Store (ODS) (Golfarelli, et al., 2004), (Sen \& Sinha, 2005), i.e., a database that gives a unified yet operational view of data. RADAR provides employees with the RADAR Data Model: this is in the middle between an ontology of concepts and terms and the actual operational (and relational) schema of source data. Once defined, the RADAR Schema (called this way because it is based on the RADAR Data Model) gives a high-level view of source data according to the concepts described in the ontology for the specific application domain. In other words, it gives a concrete view of the concepts on the basis of the actual data stored within the ODS on top of which the framework operates. Employees see the RADAR Schema, which is closer to their perception of data: they can exploit it to specify aggregated measures to put into reports, by exploiting terms coming from the ontology in order to find out how to interpret and use data. We will exploit a running example carried out in the preliminary experiments of the implemented framework, concerning data flows about loans and financing, on which a bank has to monthly prepare reports to send to central banks and rating agencies.

The paper is organized as follows: Section 2 introduces relevant related works and technical background. Section 3 describes the approach we devised; specifically, Section 3.1 introduces the RADAR Data Model, while Section 3.2 briefly introduces the RADAR Rule Language that accompanies the data model. Section 4 describes the RADAR framework and its architecture; furthermore, Section 4.1 describes the Knowledge Designer, i.e., the component devised to design the data model and to connect it to both the ontology and the data; Section 4.3 briefly presents the Report Designer, the component that allows employees to design reports. Finally, concluding remarks are reported in Section 5.

\section{BACKGROUND}

An ontology denotes the attempt to formulate an exhaustive, rigorous and hierarchically structured conceptual scheme within a given domain, which can be used as the basis of a knowledge base (Neches, et al., 1991), (Gruber, 1993).

Conceptualizing a domain corresponds to model an abstract phenomenon of the real world, by means of the explicit definition of concepts, properties and constraints (Şimşek, et al., 2017). Moreover, three categories of ontologies are identified (Swartout, et al., 1997): top-level ontologies, domain ontologies, and application ontologies, depending on the degree of abstraction of the concepts (top-level ontologies define very general concepts, while application ontologies define specific concepts of a given application domain).

In this context, several approaches have been considered and addressed in the literature. For example, the work by Berners Lee in (Berners-lee, et al., 2001) exploits ontologies to define a knowledge base by considering concepts only: neither data nor their management issues are considered. The fact that concepts are not tied to data makes this approach useless for querying data moving from the knowledge base.

In this work, the ambition of linked data is to allow for publishing data on the web in a readable and interpretable way by a machine. This should allow for defining a global data network, whose contents can be exchanged and interpreted by machines, that is, the basic idea of the semantic Web (Berners-lee, et al., 2001).

Clearly, the formats for representing ontologies play a critical role. In this sense, it is worth mentioning a W3C recommendation that specifies the JSON-LD format (Sporny, et al., 2014). JSONLD is a standard format for encoding Linked Data by using JSON as basic syntactic framework. Specifically, JSON-LD is designed around the concept of "context": it links object properties in a JSON document to concepts in an ontology. In order to map the JSON-LD format to Resource Description Framework (RDF) (Lassila, et al., 1998), JSON-LD allows values to be coerced to a specified type or to be tagged with a language. A context can be directly embedded in the JSON-LD document or put into a separate document and referenced by different documents. Then, JSON-LD appears to be a more flexible and modern approach to describe ontologies, w.r.t. the classical RDF approach. 
Among the possible fields of application of ontologies, there are, even historically, interests towards the financial market for inter organizational communication, thanks also to its international scope. Thus, it is not surprising that the Financial Industry Business Ontology (FIBO) has been designed: in fact, it specifies semantic linking between the financial concepts, as well as their descriptions (Browne, et al., 2019).

Together with FIBO, another, more recent ontology, named Schema.org, has become popular. As reported in the literature (Guha, et al., 2015), (Şimşek, et al., 2017), Schema.org is a well defined and large heterogeneous vocabulary that covers many domains. Even though the adoption of Schema.org has been increasing, the completeness for the linked data annotations is still a critical issue, mainly due to the size of the vocabulary, to the semantic inconsistency issue for some annotations, and to the lack of guidance for the users to decide which classes and properties have to be considered. To address this issue, some approaches have been presented in the literature, in order to analyze the vocabulary in real world applications (Kärle, et al., 2016), as well as in order to extend it for defining more expressive annotations.

Moreover, as reported in the literature (Palma, et al., 2013), ontologies are developed by domain experts to capture domain-specific knowledge. For example, FIBO exploits the knowledge about structure, properties and behavior of financial data. In particular, Linked Open Data have made a pool of heterogeneous collections available that have been annotated with controlled vocabulary terms from these ontologies. The analysis of these annotated linked open data could lead to discover patterns of annotations across multiple concepts (Abramowicz, et al., 2016).

Another real world application presented in the literature (Butler \& Abi-Lahoud, 2014), performed a series of studies about the approach generally followed by experts, employees and analysts involved in a financial reporting. The work aims to identify social and institutional mechanisms being applied to construct a standard common vocabulary, by using ontology-based models.

It is then possible to argue that the RADAR Data Model, defined through standard and abstract ontologies for the definition of a common vocabulary, linked to the data described by an information system, represents therefore an effective and efficient solution for reporting activities.

\section{THE APPROACH}

To understand the approach, we report some preliminary considerations that explain our vision.

- High-level view of data: looking at the actual structure of the source data coming from the feeding flow (and stored within databases), often attribute names do not clearly denote their semantics. This is the case of non-descriptive names: their meaning is known by database administrators, but they are not comprehensible by employees/analysts. In contrast, they need a high-level view of data that they can easily browse and understand.

- Conceptual characterization: semantics of data can be conceptually characterized by means of an ontology. Indeed, an ontology can provide the conceptual framework to better understanding data.

- Concrete and high-level view of data: the bridge between concepts in the ontology and actual data to analyze is provided by the RADAR Data Model. It is an object-oriented model where classes and relationships are named Concrete Classes and Concrete Relationships, respectively, since the RADAR Data Model gives a concrete view of concepts in the ontology. Analysts are provided with the RADAR Schema, that is a specific instance of the RADAR Data Model.

- Mapping RADAR Schema to Source Schema: the RADAR Schema must be mapped into the actual schema of source data. By this way, it will be possible to provide analysts with a high-level view of actual data. Clearly, not all properties defined in concrete classes are necessarily related to some attributes in the source data sets.

- Defining Update Rules: in order to complete properties that do not correspond to attributes in the source data, we introduce a language for defining Update Rules (called RADAR Rules). The goal of these rules is to define how to derive missing properties in new data.

In the in the remainder of the paper, we will make use of the following running example. Let us consider the case of a typical data flow in banks. Suppose we are bank $b_{1}$. A Cession is a contract signed by our bank $b_{1}$ with another bank $b_{2}$, by means of which $b_{1}$ has bought a loan from $b_{2}$; new cessions means that new loans have been bought by $b_{l}$. 
Each month, data flows provide new Financing States for each cession: for example, the residual debt, the number of paid installments and the number of not paid installments are reported by the monthly financial state. Finally, each month the Amortization Plan is received through the data flow, because it may have changed (for example, if the customer paid an installment, the corresponding item from its amortization plan is removed).

\subsection{RADAR Data Model}

We now introduce the RADAR Data Model. A sample RADAR Schema, concerning the running example, is reported in Figure 1: it will be used to explain concepts of the RADAR Data Model.

- Reference Ontology. The conceptual framework that provides general concepts to make concrete is the reference ontology. This ontology provides conceptual classes that describe high-level concepts. Conceptual classes are represented as gray solid-line rectangles, labeled with class names; arrows connecting conceptual classes represent specialization relationships (from a super-class to a sub-class).

- Concrete Class. Data items in the database are described by a concrete class. Such a class derives from one conceptual class defined in the reference ontology, giving a concrete view of it (by inheriting properties from the conceptual class and possibly defining new properties.) Actual data are instances of the class (a concrete class has an associated instance container).

- Concrete Sub-class. Concrete classes can have sub-classes; a sub-class can be further specialized into other sub-classes, and so on. A sub-class inherits all properties from the parent class, and can have specific properties.

- Look-up Relationship. Between root concrete classes, it is possible to define look-up relationships, denoted as $c_{1} \rightarrow c_{2}$. A look-up relationship indicates that an instance of class $c_{1}$ refers to an instance of class $c_{2}$. The presence of a look-up relationship denotes the existence of a referential relationship within data, even though, in this context, referential integrity is not explicitly maintained because we expect that it is ensured by the data-loading process.

- Property. A class has properties, denoted by a unique name and with a specific data type. Furthermore, we categorize a property either as categorical property or as measure property: categorical properties play the role of grouping properties when reports are designed; measure properties are aggregated in order to provide aggregated data into the reports.

- Key. A root concrete class has a key, i.e., a non-empty sub-set of properties whose value uniquely identifies an instance of the class. Consequently, when a look-up relationship $c_{1} \rightarrow \mathrm{c}_{2}$ is defined, it is necessary to specify the list of properties in class $c_{1}$ that must correspond to properties in the key of class $c_{2}$.

- Source Data Table and Mapping. Actual data are stored within the operational database as tables. Tables are represented as blue solid-line rectangles, labeled with table names. A solid-line arrow connects a table with a root concrete class: this means that properties in the concrete class are mapped (if possible) to attributes in the table (for properties without mapping, their value is assigned by RADAR Rules, see Section 3.2).

Finally, dashed-line arrows between tables denote the existence of referential relationships.

The RADAR Data Model is aimed to provide analysts with a high-level view of data; such a view can be concretely used to specify queries and aggregations. The RADAR Data Model is a high-level logical model: its level is higher than relational tables, but it is still logical. Nevertheless, the connection with concepts in the reference ontology is essential, in order to provide users with a conceptual view that helps find data to extract the desired information: the ontology helps in looking for and understanding data; concrete classes allow for specifying queries and aggregations.

In effect, the RADAR Data Model is subdivided in three overlapped layer: the Ontology layer describes the reference ontology; the Concrete layer describes concrete classes and concrete relationships; the Source Data layer describes data tables and mapping with concrete classes.

In Figure 1, the RADAR Schema for the running example is reported. Concrete classes are shown as thick solid line rectangles; sub-classes are connected to their parent class through grey dashed arrows; look-up relationships are represented as labeled thick orange arrows. For the sake of clarity, properties are not reported. Specifically, there are three root concrete classes, i.e., Cession, Financing State and Amortization Plan; class 
Financing State has two sub-classes, named Moody's Sate and BCE State, which report how the state is evaluated based on Moody's rules and BCE (European Central Bank) rules, respectively. Two look-up relationships are defined: Related Cession denotes that an instance of class Financing State refers to the related (instance of class) Cession; relationship Related Financing State denotes that an instance of class Amortization Plan refers to the related (instance of class) Financing State. The bottom layer is the Source Data layer: rectangles correspond to tables in the source database. Arrows connecting a concrete class to a table denote that the table is mapped to the concrete class. Notice that not necessarily all the properties in the concrete class will have a value coming from the table: such properties will receive a value by a RADAR Rule (see Section 3.2).

Figure 2 reports the properties for the three concrete classes we are discussing. Notice that properties Amount and Interest Rate are in all the three classes, because they are inherited from the same conceptual class Loan or Credit in the reference ontology. This should not be surprising for the reader: the concept of Loan or Credit in the reference ontology is very generic and has many facets: it denotes the contract (modeled by class Cession), the current state of the loan (modeled by class Financing State), and the installments to pay (modeled by class Amortization Plan). This case should explain the term Concrete Class: it gives a concrete view of a generic concept in the reference ontology.

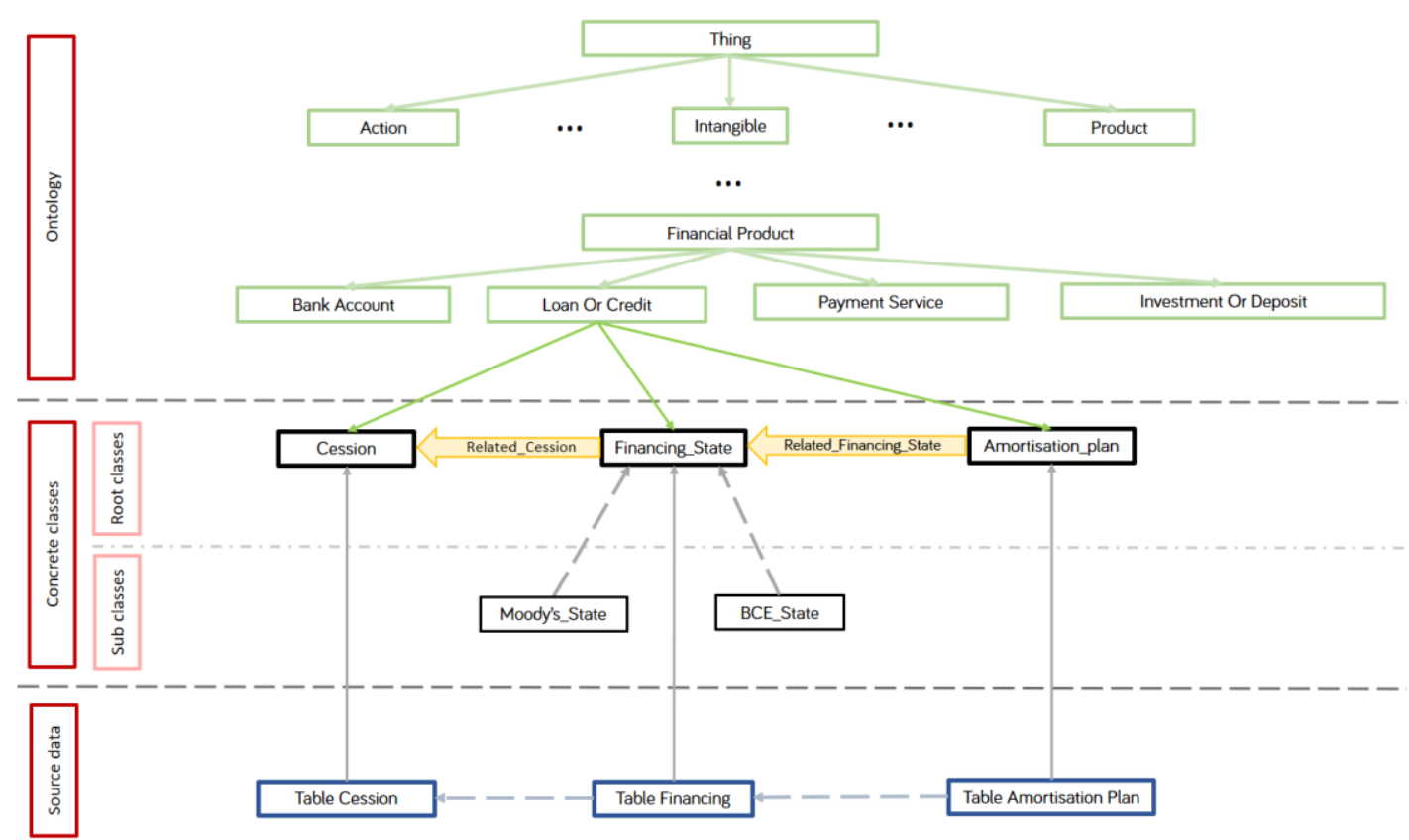

Figure 1. Graphical representation of the unified view of ontology (top), high-level logical model (middle) and source tables (bottom)

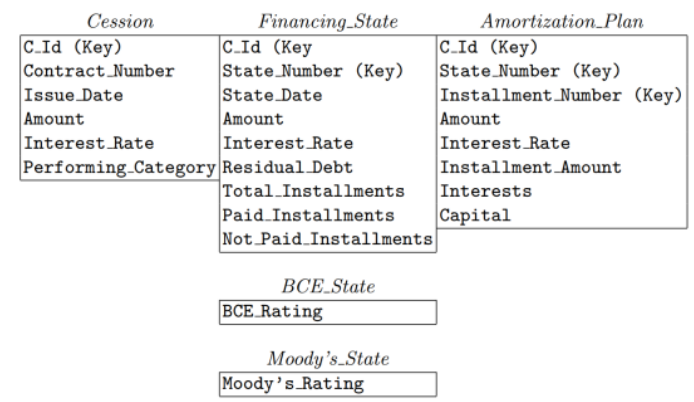

Figure 2. Properties of concrete classes reported in Figure 1 


\subsection{RADAR Rule Language}

The RADAR Data Model is completed by the RADAR Rule Language. The goal of the rule language is to provide a non-procedural way to define the values of "derived" properties, i.e., properties that are not mapped into any attribute in source tables. RADAR rules are Condition-Action (CA) rules (Zaniolo, 1994): their action is executed when the condition is true; the scope of the rule is a class instance. Their features are hereafter presented.

- Rules are associated to a concrete class defined in the RADAR Schema: they are evaluated when a new instance is stored into the database.

- The scope of the rule is one single instance: the condition is evaluated on its property values and, if true, the action is executed on it, to assign values to properties (recall that properties that are not mapped into any attribute in the source tables do not have a value; rules can be written to assign a value to these attributes).

- The relationships defined in the RADAR Schema can be used in order to get or change values of properties in related instances.

As an example, let us consider the running example and its RADAR Schema reported in Figure 1. We can define the following RADAR Rule.

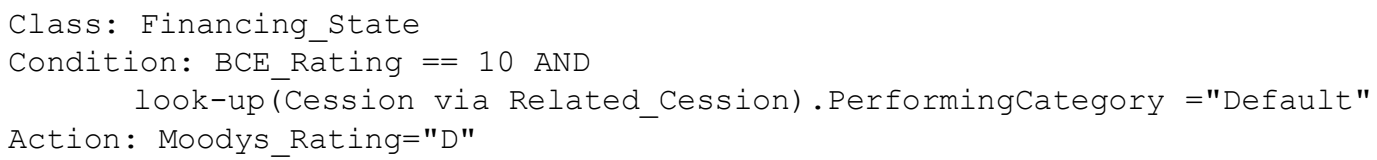

The rule is activated when a new instance of class Financing State is inserted into the database. The condition checks if the value of property BCE_Rating is 1 (that means critical) and the current state of the related cession is "Default" (notice the look-up operator, that allows for reaching the instance of class Cession by following the look-up relation Related Cession, see Figure 1). If the condition is true, the property Moody's rating is set to "D" (i.e. Default). Thus, Moody's rating is not present in the source data, but it is explicitly defined in the concrete class, with an associated description (to complete the knowledge design). Then, its value is defined by applying the rule.

\section{THE RADAR FRAMEWORK}

RADAR is a framework composed by various tools, some of them integrated within the host information system that processes the data flows. Figure 3 illustrates the overall architecture of RADAR. The dashed line separates the two layers: above the dashed line, we find the Design Layer, below it, we find the Information System Layer. Hereafter, we describe them in details.

Information System Layer: this layer contains databases, applications, services and every computational resource that constitutes the information system. As far as our framework is concerned, this layer presents the following services (the acronym $D B$ refers to DataBase term).

The Fed Source $D B$ is any kind of storage system (usually, a relational database, but now necessarily) that stores the Feeding Data Flow, i.e., those data sets that periodically enter the information system.

The Target $D B$ is the (relational) database where data to analyze must be transferred to, in order to further perform aggregations that will be the inputs for generating reports. Periodical ETL (Extract-Transform-Load) tasks should perform this transfer. This database can be seen as an Operational Data Store that provides a unified view of all feeding data sets.

The Report Generator actually generates reports, based on a Report Definition previously deployed, that rely on aggregation queries necessary to compute aggregated measures to insert into reports.

Design Layer: it is very loosely coupled to the Information System Layer, in order to overtake restrictions (properly) set by system administrators to deploy novel services into the information system. Hereafter, we present the Design Layer in details. 
The Dictionary is the own database of the RADAR framework. The name is motivated by the fact that the RADAR framework manages the reference ontology, the RADAR Schema (see Section 3.1), and the RADAR rules (see Section 3.2), all of them describing data and their corresponding terms.

- $\quad$ The Knowledge Designer is the component of the RADAR framework that provides the user interface to design the RADAR Schema (stored into the Dictionary).

A Source Ontology (or Reference Ontology) is loaded by the Knowledge Designer and saved into the Dictionary, by providing the basis for the RADAR Schema definition.

The Source-Schema Description is received from the Information System Layer, which describes data sets stored within the Fed Source DB. This description is essential to let the designer of the RADAR Schema know what data (tables and attributes/fields) are actually available in the Fed Source DB; furthermore, it is essential to drive ETL activities from Fed Source DB to Target DB.

Once the RADAR Schema is stable, it must be translated into the relational schema to deploy it into the Target DB. The schema is accompanied by Update Procedures, that power the Target DB with update procedures generated from the RADAR Rules (Section 3.2). These procedures are executed at the end of ETL on new data added to the Target DB, in order to remove incompleteness from data and, possibly, update data already stored in the Target DB.

ETL Directives are generated, in order to allow the ETL service to transfer data sets from the Fed Source DB to the Target DB.

- $\quad$ The Report Designer provides analysts with a user-friendly interface, able to browse the knowledge base so far built (reference ontology and RADAR Schema), define aggregations and design reports.

- $\quad$ Report Definitions and Aggregation Queries are deployed to the Report Generator (in the Information System Layer), to actually generate reports.

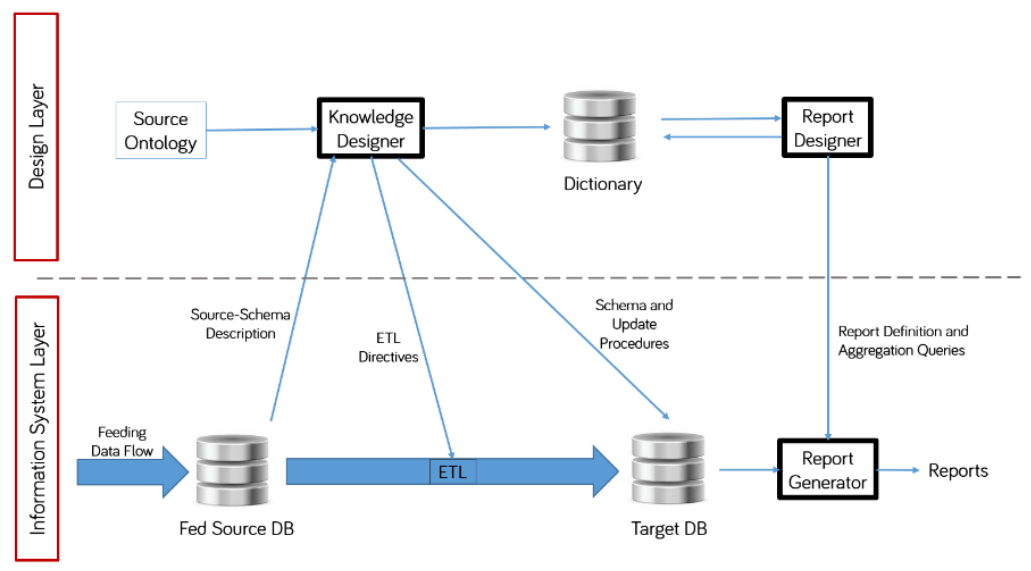

Figure 3. General Architecture of the RADAR framework

\subsection{Knowledge Designer in Details}

The Knowledge Designer is used to build the RADAR Schema and the complete knowledge base stored within the Dictionary. An example of the activities supported by the Knowledge Designer in this project is reported as follows.

1. Upload the Source Ontology that better suites the application context. In the running example concerning loans, we adopted the Schema.org ontology, because it provides the generic concepts concerning financial products.

2. Define the RADAR Schema for the specific application context. Figure 4(a) reports the screenshot of the user interface to design concrete classes. This figure shows, in the left side of the screenshot, the Ontology Browser that allows for selecting the ontological class (concept) which the new concrete class derives from. After the ontological class is selected, a window providing the tools to define the schema of the new concrete class is opened: this window (shown in the right side of the same screenshot) allows for defining the concrete class Cession, whose schema is reported in Figure 2. 
Notice that inherited properties do not appear, because they are shown in a preliminary stage, immediately after the ontological class is selected (not detailed in Figure 4(a)).

3. The RADAR Schema is completed by defining look-up relationships (see Section 3.1). Figure 4(b) shows a screenshot of the window to define the look-up relationship named Related-Cession, presented in Section 3.1 and shown in Figure 1. In particular, properties in the referring class (in this case, the Financing State class, on the left side of the screenshot) are associated to properties in the key of the referred class (in this case, the Cession class, on the right side of the screenshot).

4. Source-Schema Definitions are received from the Fed Source DB, in order to know the actual structures of data to associate with concrete classes.

5. A Mapping activity is necessary to associate concrete classes with data sets in the Fed Source DB: designers know exactly which properties are available in the source data and which must be derived.

6. After the Mapping activity is concluded, it is necessary to define the RADAR Rules (see Section 3.2) that assign values to derived properties on new data.

7. Once the RADAR Schema is stable, it is necessary to deploy it into the Information System Layer. This activity generates the Schema and Update Procedures to prepare the Target DB, as well as ETL Directives, in order to generate and deploy ETL processes that transfer data from the Fed Source DB to the Target DB.

Clearly, designing knowledge is a complex and possibly long task. Anyway, this is necessary to provide analysts with a powerful tool to define reports.

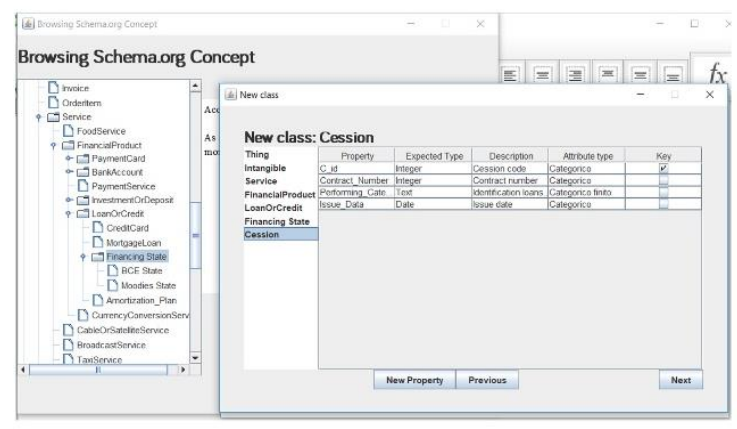

(a)

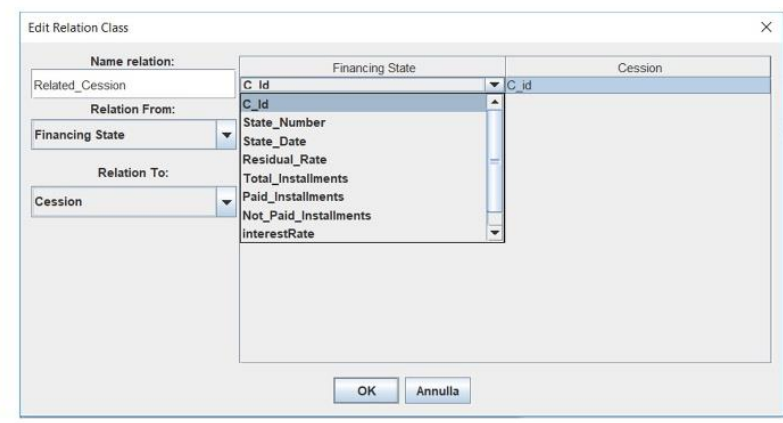

(b)

Figure 4. Screenshots of the Knowledge Designer

\subsection{Report Designer}

The Knowledge Designer is used by experts of the application domain and by experts of the database environment. They operate in the early stage of deployment of the RADAR framework. The analysts start by using the RADAR framework once the knowledge base is fully designed. By means of the Report Designer, analysts design the reports they need, by browsing the knowledge base. Hereafter, we briefly describe functionalities provided by the Report Designer.

1. The Knowledge Browser allows analysts to browse terms within the dictionary. They can consult the RADAR Schema and define the aggregations they are interested in.

2. The Report-Layout Designer allows analysts to define the layout of the reports.

3. The Deploy functionality generates Report Definitions and Aggregation Queries, to deploy into the Information System Layer. Indeed, the actual generation of reports is performed, within the Information System Layer on data stored in the Target DB, by the Report Generator component.

\section{CONCLUSIONS}

The presented approach defines and implements RADAR (Rich Advanced Design Approach for Reporting), a novel software framework that, moving from the notion of Operational Data Store, arises as a bridge between employees/analysts that need to specify how to generate a report (on the basis of concepts and terms typical of 
the application context) and the information system that stores data to query, so as to produce reports. The corresponding RADAR Data Model is, consequently, in the middle between an ontology of concepts and terms and the actual operational (and relational) schema of source data. Once defined, the RADAR Schema gives a high-level view of source data on the basis of concepts described in the ontology for the specific application context. In other words, it gives a concrete view of concepts on the basis of actual data stored within the ODS on top of which the framework works. Employees/analysts see the RADAR Schema, that is closer to their perception of data: they can exploit it to specify the aggregated measures to put into reports, by exploiting terms coming from the ontology, to find out how to interpret and use data. The approach is general, even if in this work we exploited a running example inspired by the context of financial reporting. In this paper, we focused on the Knowledge Designer, which is the most critical component to make effective the overall RADAR framework. The Report Designer is in a prototypical stage and will be presented in details in future works.

Further future work will encompass the assessment of the approach, in order to verify the effectiveness of the implemented framework, as far as all the components of the framework are concerned. In particular, the user interface of the Report Designer will be carefully evaluated and step-by-step refined, in order to provide analysts with an effective tool. Nevertheless, efficiency at query time and report generation time is crucial: this issue will be deeply investigated too. Finally, we will explore, together with finance, also other application contexts, in order to disseminate the approach to various directions (Butler \& Abi-Lahoud, 2014).

\section{REFERENCES}

Abramowicz, W., Auer, S. \& Heath, T., 2016. Linked Data in Business. web intelligence, 58(5), pp. 323-326.

Berners-lee, T., Hendler, J. A. \& Lassila, O., 2001. The Semantic Web" in Scientific American. .

Browne, O., O'Reilly, P., Hutchinson, M. \& Krdzavac, N. B., 2019. Distributed data and ontologies: An integrated semantic web architecture enabling more efficient data management. Journal of the Association for Information Science and Technology, 70(6), pp. 575-586.

Butler, T. \& Abi-Lahoud, E., 2014. A Mechanism-Based Explanation of the Institutionalization of Semantic Technologies in the Financial Industry. International Working Conference on Transfer and Diffusion of IT, pp. 277-294.

Golfarelli, M., Rizzi, S. \& Cella, I., 2004. Beyond data warehousing: what's next in business intelligence?. s.1., s.n., pp. 1-6.

Gruber, T. R., 1993. A translation approach to portable ontology specifications. Knowledge Acquisition, 5(2), pp. 199-220.

Guha, R. V., Brickley, D. \& Macbeth, S., 2015. Schema.org: evolution of structured data on the web. ACM Queue, 59(2), pp. 44-51.

Kärle, E., Fensel, A., Toma, I. \& Fensel, D., 2016. Why Are There More Hotels in Tyrol than in Austria? Analyzing Schema.org Usage in the Hotel Domain. s.l., s.n., pp. 99-112.

Lassila, O., Swick, R. R. \& W3C, C., 1998. Resource Description Framework (RDF) Model and Syntax Specification. s.l.:s.n.

Neches, R. et al., 1991. Enabling technology for knowledge sharing. Ai Magazine, 12(3), pp. 36-56.

Palma, G., Vidal, M.-E., Raschid, L. \& Thor, A., 2013. Exploiting semantics from ontologies and shared annotations to find patterns in annotated linked open data. LISC'13 Proceedings of the 3rd International Conference on Linked Science - Volume 1116, pp. 15-29.

Sen, A. \& Sinha, A. P., 2005. A comparison of data warehousing methodologies. Communications of The ACM, 48(3), pp. 79-84.

Şimşek, U., Kärle, E., Holzknecht, O. \& Fensel, D., 2017. Domain Specific Semantic Validation of Schema.org Annotations. arXiv preprint arXiv:1706.06384.

Sporny, M. et al., 2014. JSON-LD 1.0: a JSON-based serialization for linked data. W3C Recommendation, Volume 16, p. 127.

Swartout, B., Patil, R., Knight, K. \& Russ, T., 1997. Toward Distributed Use of Large-Scale Ontologies t. .

Zaniolo, C., 1994. A Unified Semantics for Active and Deductive Databases. Rules in Database Systems, pp. 271-287. 